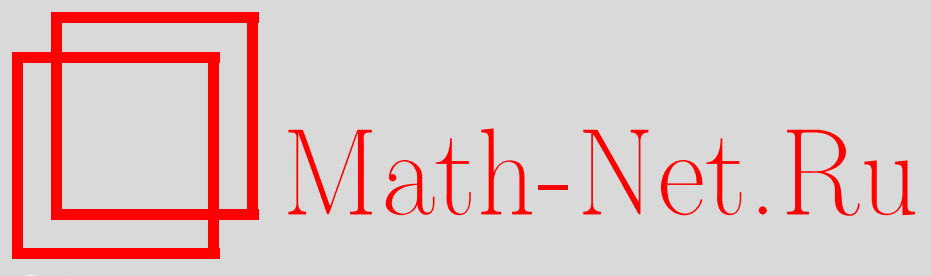

Д. Н. Иванов, Ортогональные разложения прямых сумм и тензорных произведений алгебр, УМН, 2009, том 64, выпуск 2, 205-206

DOI: https://doi.org/10.4213/rm9264

Использование Общероссийского математического портала Math-Net.Ru подразумевает, что вы прочитали и согласны с пользовательским соглашением http://www . mathnet.ru/rus/agreement

Параметры загрузки:

IP : 52.90 .164 .192

26 апреля 2023 г., 10:48:08

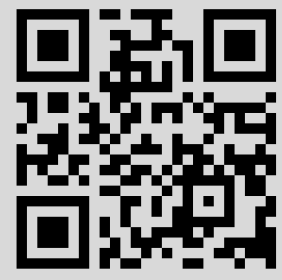




\title{
Ортогональные разложения прямых сумм и тензорных произведений алгебр
}

\author{
Д. Н. Иванов
}

Ортогональное разложение алгебры в сумму $d$-мерных коммутативных подалгебр называется $d$-разложением. В настоящей статье приводятся конструкции $d$-разложений для примарных $d$ в тензорном произведении и некоторых прямых суммах алгебр, допускающих $d$-разложения. Приведенные результаты обобщают полученные ранее для $d=2[1]$.

Напомним основные определения. Термин "алгебра" будет обозначать ассоциативную конечномерную над полем комплексных чисел $\mathbb{C}$ полупростую алгебру. Через $\operatorname{Tr}_{\mathbf{A}}$ будем обозначать след регулярного представления алгебры $\mathbf{A}$.

ОПредЕлЕниЕ. Семейство $\mathscr{D}=\left\{A_{i}, i=1, \ldots, r\right\}$ неединичных собственных подалгебр алгебры А образует ее ортогональное разложение (ОР), если все подалгебры $A_{i}$ полупросты, содержат единичный элемент $\mathbf{1}_{\mathbf{A}}$ алгебры $\mathbf{A}$ и выполняется условие ортогональности: алгебра $\mathbf{A}$ является прямой суммой попарно ортогональных относительно формы следа $\operatorname{Tr}_{\mathbf{A}} x y$ подпространств $\mathbf{A}=\left\langle\mathbf{1}_{\mathbf{A}}\right\rangle \oplus A_{1}^{\circ} \oplus \cdots \oplus A_{r}^{\circ}$, где $A_{i}^{\circ}=\left\{x \in A_{i} \mid \operatorname{Tr}_{\mathbf{A}} x=0\right\}$.

Если все подалгебры ОР $\mathscr{D}$ коммутативны, то $\mathscr{D}$ называется коммутативным. Если все подалгебры в $\mathscr{D}$ изоморфны алгебре $\mathbf{B}$, то $\mathscr{D}$ называется однородным OP muna $\mathbf{B}$ (или просто OP muna $\mathbf{B}$ ). Через $k \mathrm{M}_{n}$ будем обозначать прямую сумму $k$ экземпляров алгебры матриц $\mathrm{M}_{n}(\mathbb{C})=\mathrm{M}_{n}$. Однородное $\mathrm{OP}$ типа $d \mathrm{M}_{1} \cong \mathbb{C}^{d}$ называется d-разложением.

Отметим, что понятие однородного коммутативного ОР обобщает ряд известных понятий. Так, матрицы Адамара порядка $n$ эквивалентны 2-разложениям алгебры $n \mathrm{M}_{1}$, конечные аффинные плоскости порядка $n$ эквивалентны $n$-разложениям алгебры $n^{2} \mathrm{M}_{1}$, аффинные 2-схемы эквивалентны однородным ОР коммутативных алгебр, ортогональные разложения простых алгебр Ли типа $A_{n-1}$ в сумму картановских подалгебр эквивалентны $n$-разложениям алгебры $\mathrm{M}_{n}[1]$.

В [2] сформулирована следующая гипотеза, обобщающая известные гипотезы о порядках конечных плоскостей, о параметрах аффинных 2-схем и о простых алгебрах Ли типа $A_{n}$, допускающих ортогональные разложения в сумму картановских подалгебр.

Гипотеза 1. Если алгебра А допускает d-разложение, то $d$ есть степень простого числа. При этом если $d>2$, то $\operatorname{dim} \mathbf{A}$ является степенъю $d$.

Приведем некоторые факты о $d$-разложениях.

Теорема 1 [1]. Пусть подалгебры $\left\{A_{1}, \ldots, A_{r}\right\}$ образуют $d$-разложение алгебры $\mathbf{A}, \operatorname{dim} \mathbf{A}=n$. Пусть $\operatorname{Pr}\left(A_{s}\right)=\left\{e_{1 s}, \ldots, e_{d s}\right\}-$ примитивные идемпотенты подалгебры $A_{s}, s=\overline{1, r}$. Тогда:

1) $\operatorname{Tr}_{\mathbf{A}} e_{1 s}=\cdots=\operatorname{Tr}_{\mathbf{A}} e_{d s}=n / d$ для любого $s=\overline{1, r}$, в частности, $n$ кратно $d$;

2) элементы $\mathbf{1}_{\mathbf{A}}, e_{j s}, j=\overline{1, d-1}, s=\overline{1, r}$, образуют базис в $\mathbf{A}$, в частности, $r=(n-1) /(d-1)$.

Следующая теорема является основной.

Теорема 2. Пусть $d$ - степень простого числа. Тогда:

1) если алгебры $\mathbf{A}$ u $\mathbf{B}$ допускают d-разложение, то их тензорное произведение (над полем $\mathbb{C}) \mathbf{A} \otimes \mathbf{B}$ также допускает $d$-разложение;

2) если алгебры $\mathbf{C}_{1}, \ldots, \mathbf{C}_{d^{k}}$ имеют одинаковую размерность и допускают d-разложение, то их прямая сумма $\mathbf{C}_{1} \oplus \cdots \oplus \mathbf{C}_{d^{k}}$ также допускает d-разложение.

Работа выполнена при поддержке РФФИ (грант № 06-01-00485-а). 
ДоказАтельство. 1) Пусть $\operatorname{dim} \mathbf{A}=n, \operatorname{dim} \mathbf{B}=m, r_{1}=(n-1) /(d-1), r_{2}=$ $(m-1) /(d-1)$ и семейства подалгебр $\mathscr{D}_{1}=\left\{A_{i}, i=\overline{1, r_{1}}\right\}, \mathscr{D}_{2}=\left\{B_{j}, j=\overline{1, r_{2}}\right\}$ образуют $d$-разложения алгебр $\mathbf{A}$ и $\mathbf{B}$ соответственно. Для каждой пары подалгебр $A_{i}, B_{j}$ определим $d-1$ подалгебру в $\mathbf{A} \otimes \mathbf{B}$ следующим образом. Пусть $\operatorname{Pr}\left(A_{i}\right)=$ $\left\{e_{i 1}, \ldots, e_{i d}\right\}, \operatorname{Pr}\left(B_{j}\right)=\left\{f_{j 1}, \ldots, f_{j d}\right\}$. В силу примарности $d$ множество из $d^{2}$ попарно ортогональных идемпотентов $e_{i s} \otimes f_{j t}, s, t=\overline{1, d}$, можно рассматривать как множество точек некоторой аффинной плоскости $\pi$ порядка $d$. Каждую прямую плоскости $\pi$ отождествим с суммой идемпотентов, инцидентных этой прямой. Тогда каждый класс параллельных прямых порождает полупростую коммутативную подалгебру в $\mathbf{A} \otimes \mathbf{B}$, содержащую $\mathbf{1}_{\mathbf{A} \otimes \mathbf{B}}$ и имеющую размерность $d$. Без ограничения общности можно считать, что суммы $e_{i s} \otimes \mathbf{1}_{B}=e_{i s} \otimes f_{j 1}+\cdots+e_{i s} \otimes f_{j d}, \mathbf{1}_{A} \otimes f_{j t}=e_{i 1} \otimes f_{j t}+\cdots+e_{i d} \otimes$ $f_{j t}, s, t=\overline{1, d}$, являются прямыми плоскости $\pi$. Эти прямые образуют два класса параллельных, которые порождают подалгебры $A_{i} \otimes \mathbf{1}_{\mathbf{B}}$ и $\mathbf{1}_{\mathbf{A}} \otimes B_{j}$. Подалгебры, порождаемые оставшимися $d-1$ классами параллельных прямых, обозначим $L_{i j}^{k}$, $k=\overline{1, d-1}$. Легко проверить, что ортогональные дополнения к $\mathbf{1}_{\mathbf{A} \otimes \mathbf{B}}$ в каждой из подалгебр семейства $\mathscr{D}=\left\{A_{i} \otimes \mathbf{1}_{\mathbf{B}}, \mathbf{1}_{\mathbf{A}} \otimes B_{j}, L_{i j}^{k}, i=\overline{1, r_{1}}, j=\overline{1, r_{2}}, k=\overline{1, d-1}\right\}$ попарно ортогональны между собой. Кроме того, количество указанных подалгебр равно $r_{1}+r_{2}+(d-1) r_{1} r_{2}=(n m-1) /(d-1)=(\operatorname{dim} \mathbf{A} \otimes \mathbf{B}-1) /(d-1)$, откуда следует, что для них выполняется условие ортогональности. Значит, семейство $\mathscr{D}$ образует $d$-разложение алгебры $\mathbf{A} \otimes \mathbf{B}$.

2) Достаточно доказать утверждение в случае $k=1$. Пусть $\mathbf{C}=\mathbf{C}_{1} \oplus \cdots \oplus \mathbf{C}_{d}$, $\operatorname{dim} \mathbf{C}_{i}=n, r=(n-1) /(d-1)$ и алгебра $\mathbf{C}_{i}$ допускает $d$-разложение $\mathscr{D}_{i}=\left\{C_{i j}\right.$, $j=\overline{1, r}\}, i=\overline{1, d}$. Для каждого $j=\overline{1, r}$ определим семейство подалгебр следующим образом. Пусть $\operatorname{Pr}\left(C_{i j}\right)=\left\{e_{i s}, s=\overline{1, d}\right\}, i=\overline{1, d}$. Отождествим алгебры $\mathbf{C}_{i}$ с их каноническими образами в С. В силу примарности $d$ множество из $d^{2}$ попарно ортогональных идемпотентов $e_{i s}, i, s=\overline{1, d}$, можно рассматривать как точки некоторой аффинной плоскости $\pi$ порядка $d$. Как и раньше, каждую прямую плоскости $\pi$ отождествим с суммой идемпотентов, инцидентных этой прямой, при этом каждый класс параллельных прямых будет порождать полупростую коммутативную подалгебру в $\mathbf{C}$, содержащую $\mathbf{1}_{\mathbf{C}}$ и имеющую размерность $d$. Без ограничения общности можно считать, что суммы $\mathbf{1}_{\mathbf{C}_{i}}=e_{i 1}+\cdots+e_{i d}, i=\overline{1, d}$, являются прямыми плоскости $\pi$. Они порождают подалгебру $E=\left\langle\mathbf{1}_{\mathbf{C}_{1}}, \ldots, \mathbf{1}_{\mathbf{C}_{d}}\right\rangle$. Подалгебры, порождаемые оставшимися $d$ классами параллельных прямых, обозначим через $L_{j t}, t=\overline{1, d}$. Легко проверить, что ортогональные дополнения к $\mathbf{1}_{\mathrm{C}}$ в каждой из подалгебр семейства $\mathscr{D}=$ $\left\{E, L_{j t}, j=\overline{1, r}, t=\overline{1, d}\right\}$ попарно ортогональны. Кроме того, количество указанных подалгебр равно $1+d r=1+d(n-1) /(d-1)=(n d-1) /(d-1)=(\operatorname{dim} \mathbf{C}-1) /(d-1)$, откуда следует, что для них выполняется условие ортогональности. Значит, семейство $\mathscr{D}$ образует $d$-разложение алгебры C. Теорема доказана.

ЗАмечания. 1. Если гипотеза 1 справедлива, то ограничение на $d$ в теореме 2 можно убрать. 2. В случае $d=2$ теорема 2 в п. 2) допускает усиление: количество слагаемых $2^{k}$ можно заменить на произвольное $n$ такое, что существует матрица Адамара порядка $n$ [3]. 3. Как показано в [1], алгебра $3 \mathrm{M}_{2}$ не допускает 2-разложений, несмотря на то, что алгебры $\mathrm{M}_{2}$ и $2 \mathrm{M}_{2}$ допускают 2-разложения. Этот пример указывает на существенность условий о равенстве размерностей и о количестве слагаемых в теореме 2, п. 2).

\section{Список литературы}

[1] Д. Н. Иванов, Матем. сб., 189:12 (1998), 83-102. [2] Д. Н. Иванов, УМН, 60:2 (2005), 163-164. [3] Д. Н. Иванов, УМН, 58:4 (2003), 147-148.

Д. Н. Иванов (D. N. Ivanov)

Тверской государственный университет

E-mail: dni@tvcom.ru
Представлено А. В. Михалёвым Принято редколлегией 07.12 .2008 